\title{
Two- to Four-Year Follow-up Results of Total Knee Arthroplasty Using a New High-Flexion Prosthesis
}

\author{
Man Soo Kim, MD, In Jun Koh, MD, Sung Won Jang, MD, Neung Han Jeon, MD, and Yong In, MD \\ Department of Orthopaedic Surgery, Seoul St. Mary's Hospital, The Catholic University of Korea College of Medicine, Seoul, Korea
}

\begin{abstract}
Purpose: The purpose of this study was to evaluate minimum 2-year follow-up results of total knee arthroplasty (TKA) performed using a new highflexion prosthesis design (LOSPA).

Materials and Methods: The 2- to 4-year results of 191 consecutive TKAs (177 patients) with the LOSPA posterior-stabilized prosthesis were evaluated. The patients were assessed clinically and radiographically using the Knee Society scoring system (KSS) and the Western Ontario and McMaster Universities Osteoarthritis Index (WOMAC).

Results: The mean range of motion (ROM) increased significantly from $117.4^{\circ}$ (range, $75^{\circ}$ to $140^{\circ}$ ) preoperatively to $126.7^{\circ}$ (range, $80^{\circ}$ to $144^{\circ}$ ) postoperatively $(\mathrm{p}<0.001)$. The mean KSS and WOMAC scores improved significantly from 121.4 (range, 42 to 185 ) and 56.1 (range, 23 to 88 ) preoperatively to 174.0 (range, 130 to 200 ) and 16.4 (range, 0 to 85 ) postoperatively, respectively (both, $\mathrm{p}<0.001$ ). One knee required revision for deep infection. No knee had aseptic loosening or osteolysis. Radiolucent lines were noted in 15 knees (7.9\%).

Conclusions: The new high-flexion total knee prosthesis resulted in no early aseptic loosening of the component and improved postoperative ROM comparable to other high-flexion TKA prostheses at 2- to 4-year follow-ups.
\end{abstract}

Keywords: Knee, Arthroplasty, Range of motion, Prosthesis design, Outcome assessment

\section{Introduction}

Total knee arthroplasty (TKA) has been widely accepted as an effective treatment for end stage osteoarthritis demonstrating satisfactory outcomes and survivorship. TKA is advantageous in relieving pain and improving knee function ${ }^{1)}$. Still, restoration of normal range of motion (ROM) after TKA remains as a challenge; in general, the ROM of the knee after conventional TKA (range, $110^{\circ}$ to $120^{\circ}$ ) is less than that of a normal $\mathrm{knee}^{2)}$. In particular, squatting, sitting cross-legged, and kneeling with deep

Received September 13, 2015; Revised (1st) October 28, 2015;

(2nd) December 2, 2015; Accepted December 15, 2015

Correspondence to: Yong In, MD

Department of Orthopaedic Surgery, Seoul St. Mary's Hospital, The

Catholic University of Korea College of Medicine, 222 Banpo-daero,

Seocho-gu, Seoul 06591, Korea

Tel: +82-2-2258-2838, Fax: +82-2-535-9834

E-mail: iy1000@catholic.ac.kr

This is an Open Access article distributed under the terms of the Creative Commons Attribution Non-Commercial License (http://creativecommons.org/licenses/by-nc/4.0/) which permits unrestricted non-commercial use, distribution, and reproduction in any medium, provided the original work is properly cited. flexion of the knee joint that involve $>125^{\circ}$ of knee flexion are often required during daily living in Asian culture ${ }^{3)}$. Thus, highflexion prostheses were developed to meet such demands ${ }^{4)}$.

High-flexion TKA is designed to achieve $>125^{\circ}$ of knee flexion $^{5}$. The femoral component of the high-flexion design has a 2-4 $\mathrm{mm}$ extended and thicker posterior condyle that does not necessitate additional bone cut to maintain the contact area during deep knee flexion ${ }^{6,7)}$. The increased contact area helps posterior femoral translation. In addition, the larger posterior condyle of the femoral component decreases the risk of impingement between the posterior cortex of the femur and the polyethylene insert $^{8)}$. The new prosthesis has a modified cam and post mechanism with increased jump distance to avoid dislocation under deep flexion angles ${ }^{9)}$. It also has a longer trochlear groove for improved patellar tracking. The anterior recess in the tibial polyethylene insert reduces impingement on the patella and the patellar tendon during deep knee flexion.

The new high-flexion concept LOSPA knee system (Corentec, Seoul, Korea) has recently become available. It is designed to achieve ligament isometry from $0^{\circ}$ to $90^{\circ}$ using a single radial axis of the femoral component. The single radius of the femoral 
component contributes to stability in the mid-flexed state ${ }^{10)}$. Cam-post engagement occurs at $75^{\circ}$, which prevents unnecessary cam-post contact during level walking and has an advantage of posterior translation of the femoral condyle. The high-flexion design with more rounded femoral contour and deepened patellar groove facilitates deep flexion by reducing joint capsule overstuffing. The elongated and laterally angled patellar groove is constructed to produce good patellar tracking. This prosthesis has a $10-\mathrm{mm}$ thick posterior condyle of the femoral component that replaces the $10-\mathrm{mm}$ posterior femoral bone cut, which contributes to increased surface contact and bone support through a relatively larger posterior radius than the conventional design (Fig. 1). Compared to Nexgen LPS-flex (Zimmer, Warsaw, IN, USA), another high-flexion design implant, that requires $12-\mathrm{mm}$ posterior femoral bone cutting, LOSPA prosthesis is more advantageous for bone preservation.

Numerous studies have compared the high-flexion and conventional designs. Some studies demonstrated the superiority of the high-flexion prosthesis with regard to the postoperative ROM and function ${ }^{4,11)}$ while others showed the opposite ${ }^{7,12)}$. A concern has been raised that high-flexion TKA accelerates early aseptic loosening compared to conventional TKA due to the increased shear force between the femoral component and the femur during high flexion of the knee ${ }^{13,14)}$. Cho et al. ${ }^{13)}$ and Han et al. ${ }^{14)}$ noted a high rate of aseptic loosening of the femoral component in their patients after high-flexion TKA. In this study, we retro-

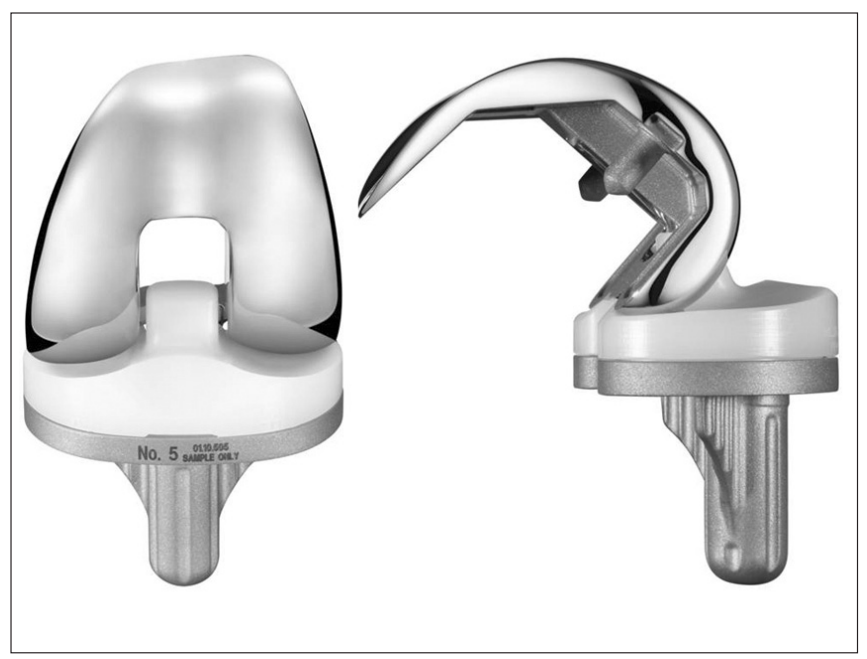

Fig. 1. Femoral component, tibial insert, and tibial component of the LOSPA total knee prosthesis. LOSPA prosthesis requires removal of additional bone from the posterior femoral condyle to add $10 \mathrm{~mm}$ posterior condyle at a large posterior radius of the femoral component. In addition, the femoral component has a more rounded contour and a deepened patellar groove to help deep flexion. The posterior surface of the insert is released for deep flexion favoring design. spectively evaluated the clinical and radiographic results of TKA performed using the newly introduced LOSPA knee system for a minimum 2-year follow-up. We examined the following two hypotheses: 1) the ROM of the knee with the LOSPA prosthesis would be comparable to that with other high-flexion concept prostheses of the recent literatures in Korean patients and 2) the implantation of the LOSPA prosthesis would be stable without aseptic loosening at minimum 2- year follow-up.

\section{Materials and Methods}

A total of 198 patients (215 knees) underwent TKA with the posterior-stabilized (PS) LOSPA total knee prosthesis from May 2011 to August 2013 at our institution. Of these, we retrospectively reviewed 191 knees of 177 patients (88\%) who were available for a minimum 2-year follow-up. Inclusion criteria were patients with indications for primary TKA: Kellgren-Lawrence classification grade 3-4 severe osteoarthritis, osteonecrosis ${ }^{15)}$, and rheumatoid arthritis. Exclusion criteria were contraindications of TKA such as knee joint infection. Patient demographics are summarized in Table 1. The mean follow-up period was 2.6 years (range, 2 to 4 years).

All surgeries were performed by a senior author (In) using the PS LOSPA knee system. All procedures were performed through a subvastus approach under general anesthesia with tourniquet inflation to $300 \mathrm{mmHg}$. All components were fixated with bone cement. The drainage tube was removed 2 days after operation. Postoperatively, none of the patients did continuous passive

Table 1. Patient Demographics

\begin{tabular}{|c|c|}
\hline Characteristic & $\begin{array}{c}\text { LOSPA TKA group } \\
(n=191 \text { knees of } 177 \text { patients) }\end{array}$ \\
\hline Gender (F:M) & $162: 15$ \\
\hline Bilateral:unilateral & $14: 163$ \\
\hline Age (yr) & $68.8 \pm 6.7(42-85)$ \\
\hline Body mass index $\left(\mathrm{kg} / \mathrm{m}^{2}\right)$ & $26.4 \pm 3.4(13.8-37.0)$ \\
\hline Operation side (R:L) & $100: 91$ \\
\hline \multicolumn{2}{|l|}{ Diagnosis } \\
\hline Osteoarthritis & 180 \\
\hline Rheumatoid arthritis & 9 \\
\hline Osteonecrosis & 2 \\
\hline Preoperative HKA axis $\left({ }^{\circ}\right)^{\text {a) }}$ & Varus $3.7 \pm 4.8$ (varus 16.8 -valgus 12.3 ) \\
\hline Follow-up period (yr) & $2.6 \pm 0.5(2.0-4.0)$ \\
\hline
\end{tabular}

Values are presented as mean \pm standard deviation or number (range). TKA: total knee arthroplasty, R: right, L: left, HKA: hip-knee-ankle.

${ }^{a}$ Data are presented as femorotibial angle. 
motion exercises, but they were encouraged to perform active exercises according to our protocol of rehabilitation. Quadricepsstrengthening exercises were started immediately after surgery and patients began walking with use of a walker on the first postoperative day. On the second postoperative day, the patients started active ROM exercises under the guidance of the medical team.

Clinical and radiographic data were evaluated at postoperative 6 weeks, 3 months, 6 months, and 1 year and then yearly thereafter. Each knee was evaluated according to the Knee Society scoring system $(\mathrm{KSS})^{16)}$ and the Western Ontario and McMaster Universities Osteoarthritis Index (WOMAC) ${ }^{17)}$ by one of the authors (Kim). At the time of each follow-up, active ROM of the knee was measured using a standard $60-\mathrm{cm}$ goniometer with the patient placed in supine position by one of the authors (Kim) who was blinded to the implant used. All complications were recorded. Weight-bearing anteroposterior, lateral, and merchant radiographs were taken at each follow-up visit. All radiographs were reviewed to assess implant position and radiolucent lines. Radiolucent lines were examined at different zones around the femoral and tibial components using the Knee Society TKA radiographic evaluation and scoring system ${ }^{18}$. All radiolucent lines were recorded and the radiographic sign of loosening was defined as the presence of a radiolucent line more than $2 \mathrm{~mm}$ in width at the cement-bone or cement-prosthesis interface ${ }^{19)}$. Implant position in relation to the limb anatomic axis and component alignment was evaluated and the presence and location of the radiolucent lines were assessed using the KSS ${ }^{18}$. Preoperative and postoperative posterior femoral condylar offsets were also compared.

Preoperative and postoperative clinical outcomes (KSS and WOMAC scores) and radiographic alignment were compared using the paired $t$-test. A p-value of $<0.05$ was considered significant, and the analysis was performed using the IBM SPSS ver. 21.0 (IBM Co., Armonk, NY, USA).

\section{Results}

The ROM at the final follow-up increased significantly compared to the preoperative ROM (Table 2). The mean KSS improved from 121.4 (range, 42 to 185 ) preoperatively to 174.0 (range, 130 to 200) at the last follow-up $(\mathrm{p}<0.001)$. The mean WOMAC score also improved from 56.1 (range, 23 to 88 ) preoperatively to 16.4 (range, 0 to 85 ) postoperatively ( $\mathrm{p}<0.001$ ) (Table 3). The mean KSS and WOMAC scores were 165.8 (range, 105 to 200) and 28.2 (range, 2 to 65), respectively, at the 6-month follow-up, 178.9 (range, 130 to 200 ) and 22.8 (range, 3 to 57), respectively, at the 1-year follow-up, 173.6 (range, 130 to 200) and 17.9 (range, 0 to 85 ), respectively, at the 2-year follow-up, and 174.2 (range, 130 to 200) and 14.8 (range, 0 to 85 ), respectively, at the 3-year follow-up.

The mean femorotibial angle was a varus of $3.7^{\circ} \pm 4.8^{\circ}$ (range, varus of $16.8^{\circ}$ to valgus of $12.3^{\circ}$ ) preoperatively and a valgus of

Table 2. Preoperative (Preop) and Postoperative (Postop) Range of Motion

\begin{tabular}{lcc}
\hline \multicolumn{1}{c}{ Characteristic } & Preop & Postop (last follow-up) \\
\hline Range of motion $\left(^{\circ}\right)$ & $117.4 \pm 12.6(75-140)$ & $126.7 \pm 9.5(80-144)$ \\
Flexion contracture $\left(^{\circ}\right)$ & $6.2 \pm 6.3(0-30)$ & $0.4 \pm 1.7(0-10)$ \\
Maximum flexion $\left(^{\circ}\right)$ & $123.7 \pm 10.5(80-140)$ & $127.1 \pm 8.6(90-144)$ \\
\hline
\end{tabular}

Values are presented as mean \pm standard deviation (range). $\mathrm{p}$-value $<0.001$.

Table 3. Preoperative (Preop) and Postoperative (Postop) Clinical Scores

\begin{tabular}{lcc}
\hline \multicolumn{1}{c}{ Characteristic } & Preop & Postop (last follow-up) \\
\hline Knee Society score & $121.4 \pm 24.3(42-185)$ & $174.0 \pm 15.5(130-200)$ \\
Pain & $24.2 \pm 9.3(0-50)$ & $46.1 \pm 5.4(20-50)$ \\
Function & $97.2 \pm 21.3(32-140)$ & $127.9 \pm 13.6(85-150)$ \\
WOMAC score & $56.1 \pm 13.7(23-88)$ & $16.4 \pm 13.7(0-85)$ \\
Pain & $11.1 \pm 3.2(4-21)$ & $1.5 \pm 2.6(0-12)$ \\
Stiffness & $4.0 \pm 1.9(0-8)$ & $1.4 \pm 1.5(0-6)$ \\
Function & $41.0 \pm 10.8(11-64)$ & $13.5 \pm 11.6(0-72)$ \\
\hline
\end{tabular}

Values are presented as mean \pm standard deviation (range). $\mathrm{p}$-value $<0.001$. WOMAC: Western Ontario and McMaster Universities.

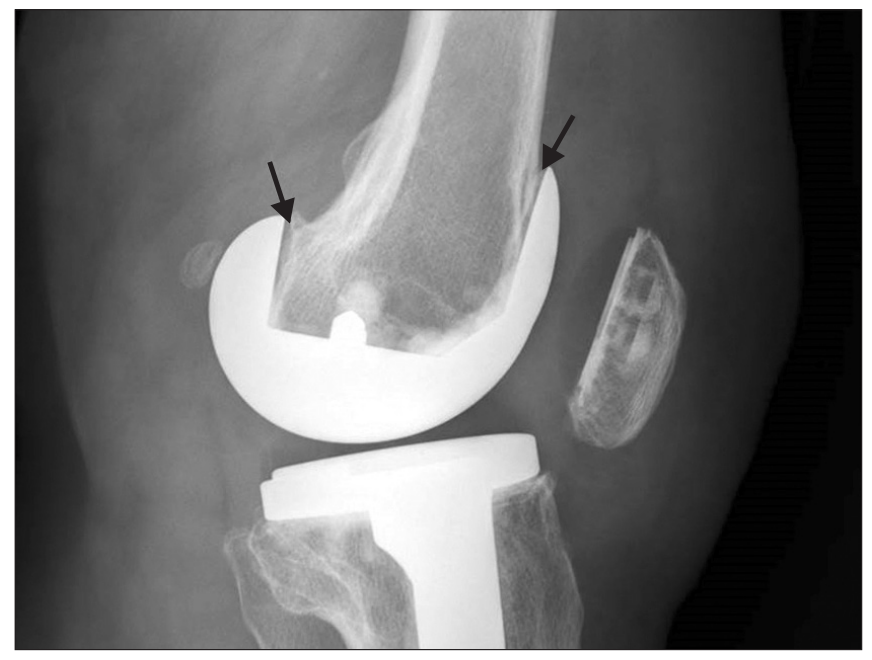

Fig. 2. The lateral radiograph of the left knee of a 68-year-old female shows radiolucent lines around the anterior cortex and posterior condylar area 3 years postoperatively. Arrow: these findings were not progressive and the patient had no symptoms or signs of aseptic loosening. 
$5.7^{\circ} \pm 1.8^{\circ}$ (range, valgus of $0^{\circ}$ to valgus of $8.4^{\circ}$ ) postoperatively $(\mathrm{p}<0.001)$. The coronal angle of the femoral component $(\alpha)$ was $96.6^{\circ} \pm 1.6^{\circ}$ (range, $92.1^{\circ}$ to $99.9^{\circ}$ ), the coronal angle of the tibial component $(\beta)$ was $89.7^{\circ} \pm 1.7^{\circ}$ (range, $84.2^{\circ}$ to $95.9^{\circ}$ ), the flexion angle of the femoral component $(\gamma)$ was $2.8^{\circ} \pm 1.3^{\circ}$ (range, $0.1^{\circ}$ to $5.5^{\circ}$ ), and the tibial slope angle $(\delta)$ was $85.8^{\circ} \pm 2.1^{\circ}$ (range, $82.1^{\circ}$ to $90.0^{\circ}$ ) postoperatively. The mean posterior femoral condylar offset was $29.6 \pm 2.5 \mathrm{~mm}$ (range, 19.8 to $36.8 \mathrm{~mm}$ ) preoperatively and $29.9 \pm 2.8 \mathrm{~mm}$ (range, 20.5 to $36.9 \mathrm{~mm}$ ) postoperatively $(\mathrm{p}=0.073)$. Radiolucent lines were found in 15 knees (14 patients, 7.9\%): two in the medial tibial condyle (zones 1 and 2), 2 in the anterior femoral condyle (zones 1 and 2), and 11 in the posterior femoral condyle (zones 3 and 4) (Fig. 2). No case of osteolysis was detected during the 2- to 4-year follow-up period. None of the knees developed aseptic loosening of the femoral, tibial, or patellar component. No knee had a tibiofemoral or patellofemoral joint dislocation.

Two major complications required surgery in two knees. A 70-year-old female with a history of lymphedema developed a deep infection in the extremity of the operated side 1 year postoperatively. The patient had received a radical hysterectomy with total lymph node dissection for uterine cervical cancer. Methicillin- sensitive Staphylococcus aureus was cultured from the joint fluid, and a two-stage revision operation was performed for the infected joint. A patellar fracture occurred in one patient and was treated with open reduction and internal fixation. Three minor complications occurred in three knees. One patient received brisement due to $<90^{\circ} \mathrm{ROM}$ limitation at 4 weeks after the operation and the final flexion angle was $125^{\circ}$. Two patients demon- strated wound dehiscence 2 weeks postoperatively. No infection was detected, and the wound was approximated with simple sutures. Symptomatic deep vein thrombosis, pulmonary embolism, and hyperextension (recurvatum) were not observed during the follow-up.

\section{Discussion}

The purpose of this study was to evaluate the ROM and early stability following TKA performed using the new high-flexion prosthesis during a short-term follow-up. The most important finding of the present study was that the newly introduced highflexion LOSPA prosthesis resulted in no early aseptic loosening of the component and provided outcomes comparable to those used in high-flexion TKA in Korean patients (Table 4).

Postoperative ROM is an important factor for patient satisfaction after TKA, particularly among Asians ${ }^{20)}$. Thus, manufacturers have modified and upgraded the design of knee prostheses to allow for high flexion after TKA. Many studies have investigated the efficacy and safety of TKA using a high-flexion design prosthesis performed by Korean surgeons, ${ }^{7,11,13,14,21-25)}$. However, there has been some puzzling inconsistency among studies on the efficacy of high-flexion TKA designs ${ }^{4,7,11,12}$. There is still a paucity of data on the ROM of the knee following TKA using this new highflexion implant; therefore, we investigated postoperative ROM in patients who received the newly designed high-flexion prosthesis. The LOSPA knee system demonstrated improved clinical and radiographic outcomes at 2- to 4-year follow-up. The outcomes of our study were comparable to those reported by other Korean

Table 4. Summary of Previous Studies on Flexion Angle, Radiolucent Lines, and Aseptic Loosening after Total Knee Arthroplasty Using High Flexion Design Prostheses

\begin{tabular}{|c|c|c|c|c|c|c|c|c|c|c|}
\hline No. & Author & $\begin{array}{l}\text { Age } \\
(\mathrm{yr})\end{array}$ & $\begin{array}{l}\mathrm{F} / \mathrm{U} \\
(\mathrm{yr})\end{array}$ & $\begin{array}{l}\text { No. of } \\
\text { knees }\end{array}$ & Implant type & $\begin{array}{c}\text { Preop } \\
\text { flexion }\left(^{\circ}\right)\end{array}$ & $\begin{array}{c}\text { Postop } \\
\text { flexion }\left(^{\circ}\right)\end{array}$ & $\begin{array}{c}\text { Radiolucent } \\
\text { lines (\%) }\end{array}$ & $\begin{array}{c}\text { Aseptic } \\
\text { loosening (\%) }\end{array}$ & $\begin{array}{l}\text { Survival } \\
\text { rate (\%) }\end{array}$ \\
\hline 1 & $\begin{array}{l}\text { Kim et al. } \\
\text { (current study) }\end{array}$ & 68.8 & 2.6 & 191 & LOSPA & 117.4 & 126.7 & 7.8 & 0 & 99.4 \\
\hline 2 & Kim et al. ${ }^{7)}$ & 68.0 & 2.1 & 50 & NexGen LPS-Flex & 127.0 & 139.0 & 0 & 0 & 100 \\
\hline 3 & Bin et al. ${ }^{11)}$ & 66.6 & 1.0 & 90 & NexGen LPS-Flex & 123.0 & 130.0 & 0 & 0 & 100 \\
\hline 4 & Cho et al. ${ }^{13)}$ & 64.0 & 4.3 & 218 & NexGen LPS-Flex & 109.0 & 129.0 & 13.8 & 3.2 & 96.8 \\
\hline 5 & Han et al. ${ }^{14)}$ & 68.3 & 2.7 & 72 & NexGen LPS-Flex & 121.0 & 132.0 & 38.0 & 21.0 & 79.0 \\
\hline 6 & Han et al. ${ }^{21)}$ & 68.3 & 7.7 & 72 & NexGen LPS-Flex & 121.0 & 132.0 & 50.0 & 46.0 & 52.0 \\
\hline 7 & Choi et al. ${ }^{22)}$ & 71.1 & 2.3 & 85 & PFC RP-F & 126.0 & 128.0 & 0 & 0 & 0 \\
\hline 8 & Seon et al. ${ }^{23)}$ & 69.1 & 2.3 & 47 & NexGen LPS-Flex & 129.0 & 130.0 & NR & NR & NR \\
\hline 9 & Lee et $\mathrm{al}^{24)}$ & 68.0 & 4.8 & 698 & NexGen LPS-Flex & 130.0 & 133.0 & 0.9 & 0.9 & 99.1 \\
\hline 10 & Kim et al. ${ }^{25)}$ & 69.9 & 3.8 & 278 & NexGen LPS-Flex & 117.3 & 135.0 & 0.4 & 0 & 99.6 \\
\hline
\end{tabular}

F/U: follow-up, Preop: preoperative, Postop: postoperative, NR: no report. 
studies on high-flexion TKA. Seo et al. ${ }^{26)}$ compared the LOSPA PS total knee prosthesis with another high-flexion PS prosthesis. In their study, the mean postoperative flexion angle of the LOSPA group was $126.9^{\circ}$. The mean postoperative ROM of the patients was similar to that of the current study. Theoretically, a knee flexion angle of $140^{\circ}$ is possible with the high-flexion LOSPA system, but it appears not to be attainable in vivo.

In the present study, the postoperative ROM of the knee was less than our expectation (up to $140^{\circ}$ ) and the superiority of the prosthesis compared with conventional TKA in terms of ROM was not observed ${ }^{7,11,22}$. Several factors may contribute to the ROM after TKA including patient factors (preoperative ROM, obesity, postoperative quality of rehabilitation, patient motivation, and preoperative mental illness), intraoperative technical issues (resection of back osteophytes, ligament balancing, component positioning, altered joint line, and posterior tibial slope), and perioperative complications $\mathrm{s}^{27}$. It has been generally considered that the preoperative ROM is the most predictable factor for the postoperative $\mathrm{ROM}^{28)}$. Our study showed that the new highflexion LOSPA prosthesis would not improve the postoperative $\mathrm{ROM}$ in patients with a preoperative ROM of $<120^{\circ}$. A controversy still exists regarding the relationship between the posterior condylar offset and the $\mathrm{ROM}^{6,29)}$. Bellemans et al. ${ }^{6}$ reported that the final knee flexion angle was significantly correlated with the posterior femoral condylar offset in TKA patients. They insisted that restoring posterior condylar offset would be very important to achieve a greater flexion angle before impingement of the posterior tibial insert against the posterior femur. In contrast, Hanratty et al. ${ }^{29)}$ found no correlation between the range of knee flexion and posterior condylar offset after TKA. In our study, postoperative posterior condylar offset was well restored compared with the preoperative value. An additional 2-mm posterior femoral bone resection was not necessary because of the $2 \mathrm{~mm}$ extension of the posterior condyle of the femoral component. Consequentially, there was no difference in the postoperative posterior condylar offset. Therefore, we found it difficult to determine the relationship between the posterior condylar offset and knee the flexion angle in the present study.

Concerns have been raised about aseptic loosening of the highflexion knee systems during the early postoperative phase. Cho et al. ${ }^{13)}$ reported that progressive radiolucent lines around the femoral component were detected in $13.8 \%$ of 218 knees that received the NexGen LPS-flex TKA at a mean follow-up of 51 months. In their study, radiolucent lines were observed around the anterior and posterior flanges of the femoral component. Han et al. ${ }^{14)}$ noted aseptic loosening in 38\% of cases at a mean follow-up of
32 months after using the same high-flexion total knee prosthesis and performed revision in $21 \%$ of cases at a mean of 23 months postoperatively. They reported radiolucent lines were most common in the anterior flange of the femoral component. In highflexion TKA, the tibial insert only articulates with the femoral component, resulting in the absence of load sharing effect and application of greater shearing force on the femoral component. Conventional TKA requires a distinctly higher loosening force compared to high-flexion TKA. Among the high-flexion designs, those with an open geometry of the internal femoral component are more vulnerable to loosening than those with a closed geometry. Peg fixation is essential to good fixation of a component and provides stability against the loosening force ${ }^{30)}$. The LOSPA total knee system requires a $10-\mathrm{mm}$ posterior femoral condylar bone cut, which is more conservative compared to the $12 \mathrm{~mm}$ required by the Nexgen LPS-flex system. In addition, peg fixation of the LOSPA prosthesis provides resistance to the loosening of component, but open femoral component geometry is not beneficial for prevention of loosening ${ }^{30)}$. Our results showed a $7.8 \%$ incidence of radiolucent lines around the anterior flange of the femur, the posterior femoral condyle, and the medial tibial condylar area. However, no symptoms or signs of aseptic loosening were detected in patients with radiolucent lines. Han et al. ${ }^{14)}$ reported that the mean flexion angle was $136^{\circ}$ in the loosening group and $125^{\circ}$ in the well- fixed group. The proportion of patients who were able to perform high-flexion activities such as squatting and kneeling was also higher in the loosening group. The femur contacted with the posterior end of tibia at $133^{\circ}$ of flexion angle. In our study, the mean flexion angle of the knee was $127^{\circ}$, which was similar with the angle of the well-fixed group and below $133^{\circ 14)}$. We did not thoroughly investigate the percentage of patients who were able to perform high flexion activities, but we recommended the patients not to be involved in high-flexion activities as much as possible. It was an attempt to prevent early loosening of component following high-flexion TKA ${ }^{13)}$. The knees with appearance of radiolucent lines require further follow-up to assure the clinical course.

Our study has some limitations. First, this was a case series, not a comparative study. We did not have a control group to compare the knee ROM and radiographic and clinical outcomes. Second, the minimum 2-year follow-up period was relatively short, so we cannot determine the long-term clinical and radiographic outcomes. 


\section{Conclusions}

The evaluation of patients who underwent TKA with the highflexion LOSPA prosthesis revealed improved clinical and radiographic results and low complication rates after a minimum 2-year follow-up. However, a long-term follow-up is required to determine whether the new high-flexion concept design demonstrates a comparable survivorship. In conclusion, the new highflexion total knee prosthesis resulted in no early aseptic loosening of the component and improved ROM comparable to other highflexion TKA prostheses at 2- to 4-year follow-up.

\section{Conflict of Interest}

No potential conflict of interest relevant to this article was reported.

\section{References}

1. Heiberg K, Bruun-Olsen V, Mengshoel AM. Pain and recovery of physical functioning nine months after total knee arthroplasty. J Rehabil Med. 2010;42:614-9.

2. Kurosaka M, Yoshiya S, Mizuno K, Yamamoto T. Maximizing flexion after total knee arthroplasty: the need and the pitfalls. J Arthroplasty. 2002;17(4 Suppl 1):59-62.

3. Mulholland SJ, Wyss UP. Activities of daily living in nonWestern cultures: range of motion requirements for hip and knee joint implants. Int J Rehabil Res. 2001;24:191-8.

4. Gupta SK, Ranawat AS, Shah V, Zikria BA, Zikria JF, Ranawat CS. The P.F.C. sigma RP-F TKA designed for improved performance: a matched-pair study. Orthopedics. 2006;29(9 Suppl):S49-52.

5. Jones RE. High-flexion rotating-platform knees: rationale, design, and patient selection. Orthopedics. 2006;29(9 Suppl):S76-9.

6. Bellemans J, Banks S, Victor J, Vandenneucker H, Moemans A. Fluoroscopic analysis of the kinematics of deep flexion in total knee arthroplasty: influence of posterior condylar offset. J Bone Joint Surg Br. 2002;84:50-3.

7. Kim YH, Sohn KS, Kim JS. Range of motion of standard and high-flexion posterior stabilized total knee prostheses: a prospective, randomized study. J Bone Joint Surg Am. 2005;87:1470-5.

8. Argenson JN, Scuderi GR, Komistek RD, Scott WN, Kelly MA, Aubaniac JM. In vivo kinematic evaluation and design considerations related to high flexion in total knee arthro- plasty. J Biomech. 2005;38:277-84.

9. Jain S, Pathak AC, Kanniyan K, Kulkarni S, Tawar S, Mane P. High-flexion posterior-stabilized total knee prosthesis: is it worth the hype? Knee Surg Relat Res. 2013;25:100-5.

10. Wang H, Simpson KJ, Ferrara MS, Chamnongkich S, Kinsey T, Mahoney OM. Biomechanical differences exhibited during sit-to-stand between total knee arthroplasty designs of varying radii. J Arthroplasty. 2006;21:1193-9.

11. Bin SI, Nam TS. Early results of high-flex total knee arthroplasty: comparison study at 1 year after surgery. Knee Surg Sports Traumatol Arthrosc. 2007;15:350-5.

12. McCalden RW, MacDonald SJ, Bourne RB, Marr JT. A randomized controlled trial comparing "high-flex" vs "standard" posterior cruciate substituting polyethylene tibial inserts in total knee arthroplasty. J Arthroplasty. 2009;24(6 Suppl):33-8.

13. Cho SD, Youm YS, Park KB. Three- to six-year follow-up results after high-flexion total knee arthroplasty: can we allow passive deep knee bending? Knee Surg Sports Traumatol Arthrosc. 2011;19:899-903.

14. Han HS, Kang SB, Yoon KS. High incidence of loosening of the femoral component in legacy posterior stabilised-flex total knee replacement. J Bone Joint Surg Br. 2007;89:1457-61.

15. Kellgren JH, Lawrence JS. Radiological assessment of osteoarthrosis. Ann Rheum Dis. 1957;16:494-502.

16. Insall JN, Dorr LD, Scott RD, Scott WN. Rationale of the Knee Society clinical rating system. Clin Orthop Relat Res. 1989;(248):13-4.

17. Bellamy N, Buchanan WW, Goldsmith CH, Campbell J, Stitt LW. Validation study of WOMAC: a health status instrument for measuring clinically important patient relevant outcomes to antirheumatic drug therapy in patients with osteoarthritis of the hip or knee. J Rheumatol. 1988;15:1833-40.

18. Ewald FC. The Knee Society total knee arthroplasty roentgenographic evaluation and scoring system. Clin Orthop Relat Res. 1989;(248):9-12.

19. Gonzalez MH, Mekhail AO. The failed total knee arthroplasty: evaluation and etiology. J Am Acad Orthop Surg. 2004;12:436-46.

20. Park KK, Shin KS, Chang CB, Kim SJ, Kim TK. Functional disabilities and issues of concern in female Asian patients before TKA. Clin Orthop Relat Res. 2007;461:143-52.

21. Han HS, Kang SB. Brief followup report: does high-flexion total knee arthroplasty allow deep flexion safely in Asian patients? Clin Orthop Relat Res. 2013;471:1492-7.

22. Choi WC, Lee S, Seong SC, Jung JH, Lee MC. Comparison 
between standard and high-flexion posterior-stabilized rotating-platform mobile-bearing total knee arthroplasties: a randomized controlled study. J Bone Joint Surg Am. 2010;92:2634-42.

23. Seon JK, Park JK, Shin YJ, Seo HY, Lee KB, Song EK. Comparisons of kinematics and range of motion in high-flexion total knee arthroplasty: cruciate retaining vs. substituting designs. Knee Surg Sports Traumatol Arthrosc. 2011;19:201622.

24. Lee BS, Chung JW, Kim JM, Kim KA, Bin SI. High-flexion prosthesis improves function of TKA in Asian patients without decreasing early survivorship. Clin Orthop Relat Res. 2013;471:1504-11.

25. Kim TH, Lee DH, Bin SI. The NexGen LPS-flex to the knee prosthesis at a minimum of three years. J Bone Joint Surg Br. 2008;90:1304-10.

26. Seo JG, Moon YW, Chang MJ, Jo BC, Park YB, Lim DS, Lee $\mathrm{BH}$. Design modifications of high-flexion TKA do not im- prove short term clinical and radiographic outcomes. BMC Musculoskelet Disord. 2014;15:433.

27. Dennis DA, Komistek RD, Scuderi GR, Zingde S. Factors affecting flexion after total knee arthroplasty. Clin Orthop Relat Res. 2007;464:53-60.

28. Ritter MA, Harty LD, Davis KE, Meding JB, Berend ME. Predicting range of motion after total knee arthroplasty. Clustering, log-linear regression, and regression tree analysis. J Bone Joint Surg Am. 2003;85:1278-85.

29. Hanratty BM, Thompson NW, Wilson RK, Beverland DE. The influence of posterior condylar offset on knee flexion after total knee replacement using a cruciate-sacrificing mobile-bearing implant. J Bone Joint Surg Br. 2007;89:915-8.

30. Bollars P, Luyckx JP, Innocenti B, Labey L, Victor J, Bellemans J. Femoral component loosening in high-flexion total knee replacement: an in vitro comparison of highflexion versus conventional designs. J Bone Joint Surg Br. 2011;93:1355-61. 